\title{
Applying new technologies for diagnosing sexually transmitted infections in resource-poor settings
}

\author{
Rosanna W Peeling
}

\section{Correspondence to}

Professor Rosanna W Peeling, London School of Hygiene and Tropical Medicine, Keppel Street, London, UK; rosanna.peeling@Ishtm.ac.uk

Accepted 24 June 2011

\section{(D) UNOCKID}

This paper is freely available online under the BMJ Journals unlocked scheme, see http://sti. bmi.com/site/about/unlocked. xhtml

\section{INTRODUCTION}

Bacterial sexually transmitted infections (STI) syphilis, chlamydia and gonorrhoea impose a disproportionate disease burden on women and children in the developing world. Diagnostic testing is particularly critical for the detection of STI, as most infected individuals have few or no symptoms. Genital chlamydial and gonococcal infections in women can lead to chronic pelvic pain, ectopic pregnancy or tubal infertility. Syphilis in pregnancy can result in stillbirth, spontaneous abortion or babies with congenital syphilis, $50 \%$ of whom may die in the first year of life. Bacterial STI are curable and treatment is affordable, but sensitive diagnostic tests are required for early detection to guide treatment to prevent the development of reproductive sequelae and adverse outcomes of pregnancy, and to interrupt onward transmission.

In resource-poor settings, laboratory services for STI are either not available, or where limited services are available, patients may not be able to pay for or physically access these services. In subSaharan Africa, only 30\% of pregnant women with syphilis are screened and treated, even though universal screening of pregnant women for syphilis is the recommended policy in many countries. The major obstacle is the lack of access to laboratories that can offer screening as women often have to travel long distances to reach a hospital or clinic with such services. Many women also fail to return for test results and treatment after the initial visit. In recent years, rapid tests have made it possible to increase access to STI testing to all levels of the healthcare system. A mathematical model estimated that a test for syphilis that requires no laboratory infrastructure could save more than 201000 lives and avert 215000 stillbirths per year worldwide. A similar test could save approximately four million disability-adjusted life-years, avert more than 16.5 million incident gonorrhoea and chlamydial infections and prevent more than 212000 HIV infections per year. ${ }^{1}$

This article examines new technologies available on a 2-4 year horizon and how they may be applied to the diagnosis of STI in resource-poor settings to reduce the burden of STI (table 1).

\section{NEW TECHNOLOGIES \\ Rapid tests}

Immunochromatographic tests (ICT) that can use fingerprick blood specimens are commercially available for HIV and syphilis. They fulfil the WHO ASSURED criteria of being affordable, sensitive, specific, user-friendly requiring minimal trained personnel, rapid and robust (no special storage conditions and can give results in $15-20 \mathrm{~min}$ ), equipment free and deliverable to those who need them. ${ }^{2}$ These rapid tests have improved the effectiveness of prevention of mother to child transmission programmes for HIV and syphilis because of shorter patient wait times, ease of use, lower cost and obviating the need for follow-up and treatment. It is now possible to develop a comprehensive rapid diagnostic package for prenatal care by combining them with rapid tests for anaemia or malaria. Rapid tests are also useful in reducing the reservoir of transmission for STI if used for screening for high-risk populations in outreach settings.

On the horizon are lateral flow ICT that are designed for duplex (HIV and syphilis) or multiplex testing (HIV, syphilis and hepatitis B or C) using a single fingerprick blood sample. Several of these tests are already in clinical trials. Details of a rapid test that can detect both non-treponemal and treponemal antibodies using patented dual path technology have recently been published. ${ }^{3}$

To improve test sensitivity, a number of simple inexpensive optical or chemiluminescent readers are now commercially available for use with ICT. The use of these readers removes observer bias, improves sensitivity and allows for quantitation such as for non-treponemal titres. Several companies are developing oral fluid tests for syphilis alone or in a duplex test with HIV.

While ICT can detect antibodies with high sensitivity, they tend to be less sensitive for the detection of antigen. Most rapid tests for the detection of genital chlamydial and gonococcal infections have sensitivities of only $50-70 \%$ compared with nucleic acid amplified tests. A recent publication reported a rapid test that detects the chlamydial lipopolysaccharide with a sensitivity of $84 \%$ and a specificity of $99 \%$ in women compared with urine PCR. ${ }^{4}$ Although promising, its utility in resource-poor settings remains unclear until independent evaluations of its performance are carried out.

\section{Microfluidic assays}

Microfluidic assays that can detect multiple analytes from a single specimen or can reproduce all the performance characteristics of an immunoassay have been developed and applied to HIV and syphilis. ${ }^{5}$ The assays have few moving parts and the chips cost pennies instead of dollars, making them suitable for resource-poor settings. The results can be read with a small battery-powered device. Some of these assays are now in trials in Africa.

\section{Molecular tests}

Recent developments in integrated nucleic acid amplification platforms have given rise to sensitive, 
Table 1 Diagnostics for major STI syndromes

\begin{tabular}{|c|c|c|c|}
\hline Major STI syndromes & Pathogens & Existing technologies & New technologies \\
\hline Urethral discharge & $\begin{array}{l}\text { Chlamydia trachomatis } \\
\text { Neisseria gonorrhoeae }\end{array}$ & $\begin{array}{l}\text { Antigen detection: enzyme immunoassay } \\
\text { or rapid antigen test } \\
N A A T\end{array}$ & $\begin{array}{l}\text { Rapid multiplex NAATs: } \\
\text { GeneXpert, } \\
\text { Recombinase Polymerase Amplification, } \\
\text { Rolling Circle Amplification } \\
\text { and other rapid NAATs }\end{array}$ \\
\hline Vaginal discharge & $\begin{array}{l}\text { Chlamydia trachomatis, } \\
\text { Neisseria gonorrhoeae } \\
\text { Trichomonas vaginalis } \\
\text { Bacterial vaginosis }\end{array}$ & $\begin{array}{l}\text { Antigen detection: enzyme immunoassay } \\
\text { or rapid antigen test } \\
N A A T \\
\text { Microscopy }\end{array}$ & $\begin{array}{l}\text { Rapid multiplex NAATs: } \\
\text { GeneXpert, } \\
\text { Recombinase polymerase amplification, } \\
\text { Rolling circle amplification } \\
\text { and other rapid NAATs }\end{array}$ \\
\hline \multirow[t]{3}{*}{ Genital ulcer } & Haemophilus ducreyi & Culture & If ulcer present: Rapid multiplex NAAT \\
\hline & Herpes simplex type 2 & Serology, NAAT & $\begin{array}{l}\text { Serology: Rapid multiplex testing, or in combination with } \\
\text { HIV, syphilis and/or Hepatitis B or C }\end{array}$ \\
\hline & Treponema pallidum & $\begin{array}{l}\text { Dark field microscopy } \\
\text { Serology: } \\
\text { Non-treponemal tests: RPR/VDRL } \\
\text { Treponemal tests: } \\
\text { TPHA/TPPA/Rapid tests }\end{array}$ & $\begin{array}{l}\text { Serology } \\
\text { Rapid duplex non-treponemal and treponemal tests } \\
\text { Rapid treponemal tests using oral fluid }\end{array}$ \\
\hline Genital warts & Human papilloma virus & $\begin{array}{l}\text { Rapid antigen detection } \\
\text { NAAT }\end{array}$ & Rapid NAAT \\
\hline
\end{tabular}

NAAT, nucleic acid amplified test; RPR, rapid plasma reagin; STI, sexually transmitted infection; TPHA, Treponema pallidum haemagglutination assay; TPPA, Treponema pallidum Particle Agglutination assay; VDRL, Venereal Disease Research Laboratory.

specific, user-friendly, sample-in, answer-out diagnostic tools for infectious diseases. These technologies are promising for the multiplex detection of pathogens associated with the major STI syndromes. The GeneXpert assay for tuberculosis has a reported sensitivity of $95 \%$ and a specificity of $98 \%$ against culture and $\mathrm{PCR}$, and can give a result for both the detection of tuberculosis and rifampicin resistance in under $2 \mathrm{~h}^{6}{ }^{6}$ Tests for chlamydia and gonorrhoea using this platform will soon be commercially available. The machine allows random access, which makes it more cost-effective if the equipment can be used for different infectious diseases. As donor agencies are planning to purchase this equipment for tuberculosis control programmes, STI programmes may be able to benefit from these investments to make this an affordable technology for resource-poor settings for the diagnosis of STI.

There are a few promising isothermal nucleic acid amplification technologies that are rapid and simple to perform. The recombinase polymerase amplification is a 15-min assay that can achieve the same sensitivity and specificity as current molecular assays by coupling isothermal recombinase-driven primer targeting of template material with strand-displacement DNA synthesis. It achieves exponential amplification with no need for pretreatment of sample DNA. ${ }^{7}$ The results can be read on a fluorimeter or in a lateral flow strip. An integrated platform for rolling circle amplification and circle-to-circle amplification and subsequent electrophoretic detection of a specific gene on a poly(methyl methacrylate) microchip has been developed. ${ }^{8}$ Rolling circle amplification and circle-to-circle amplification are carried out at $37^{\circ} \mathrm{C}$ in the sample well of the microchip, and provide a sensitive, fast, high-throughput and reproducible method for signal amplification. These are being developed for the detection of infectious disease, including chlamydia and gonorrhoea.

Rapid advances in sequencing technologies, such as nanopore technology, have made it possible to conduct genome-wide scans rapidly and at a relatively low cost. These technologies can be applied to the detection of gonococcal resistance.

\section{IMPLEMENTING NEW TECHNOLOGIES IN RESOURCE-POOR SETTINGS}

Having new technologies is only one side of the coin. ${ }^{9}$ For the best diagnostics to have the desired impact, they must reach those who are in greatest need. The 2004 Health Development Report cited lack of access and unaffordability as two major reasons why services fail. ${ }^{10}$ Access to high quality diagnostics depends on a functioning healthcare infrastructure with health workers trained and motivated to do the work. In resource-poor settings, limited laboratory facilities, few skilled laboratory personnel, unreliable supply chain management of drugs, diagnostics and other commodities, disjointed health information systems, competing vertical 'global health' initiatives, scarce drug supply and weak policy all contribute to the problems of effective implementation.

To address these problems, investments in technological innovation must be balanced with investments to strengthen health systems. ${ }^{11}$ Quality assurance programmes, developed primarily for laboratories, must be adapted for point-of-care testing. For individuals infected with STI, stigma and social norms add another layer onto physical and financial barriers to accessing diagnostic services. Implementation research on the introduction of rapid syphilis tests in China showed that those who are at highest risk of syphilis and have the largest number of clients are least likely to accept free screening and treatment for syphilis. More needs to be done to increase the uptake of screening for HIV and STI in high-risk populations.

In a recent project to introduce rapid syphilis tests to increase access to syphilis screening in prenatal and high-risk populations in seven countries in Africa, Asia and the Americas, a framework and a critical path were developed for test introduction to ensure sustainable adoption (figure 1). The framework uses a same day testing and treatment strategy and starts with political commitment, policy and planning followed by technical guidance, quality assurance and monitoring and evaluation. Political commitment for syphilis screening came from the global strategy for the prevention and control of STI, developed by the WHO for 2006-15, which includes expanding access to STI care and elimination of congenital syphilis. PAHO and Unicef have advocated for a syphilis-free and an HIV-free generation.

To facilitate more effective implementation, it would be useful to develop a tool kit for test introduction, which may consist of: a template for advocacy; a policy platform to facilitate the translation of research evidence into policy and practice; a programme managers' guide; a mathematical model to estimate the impact and cost-effectiveness of antenatal screening for 


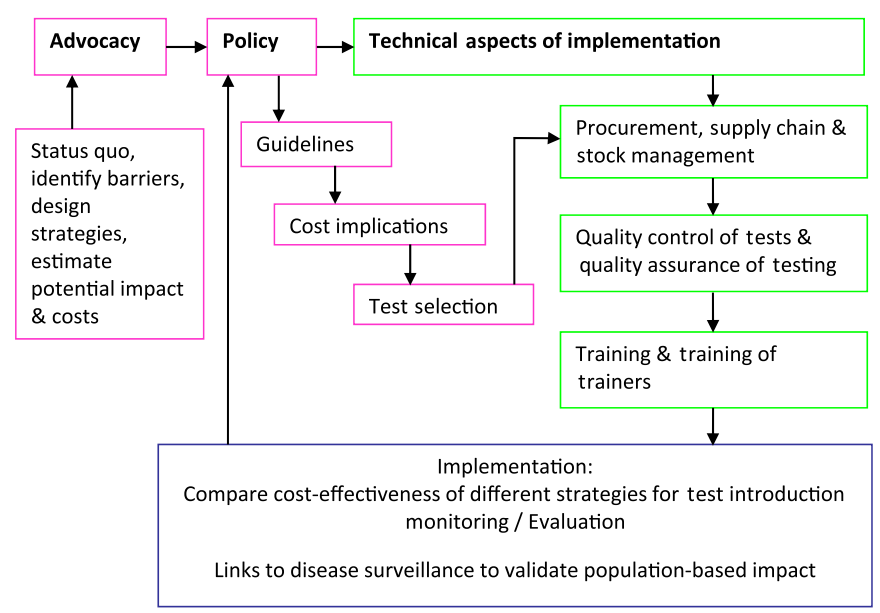

Figure 1 Draft framework for rapid sexually transmitted infection test introduction.

syphilis; a data collection tool to identify barriers to screening; testing and treatment algorithms; procurement advice and guidance on supply chain and stock management to prevent stock-outs; training and training-of-trainers packages; quality assurance/quality control systems to ensure the quality of tests and of testing; monitoring and evaluation tools for monitoring the effectiveness of training and sustainability of test introduction.

Accessible tests have the potential to empower health providers, including midwives and nurses at all sites, as they can offer a better service to their patients by screening at their first visit and giving their results immediately, rather than having to return for them. These tests can strengthen health systems by simplifying services and facilitating the integration of HIV and STI services.

\section{CONCLUSION}

Recent investments in rapid amplification and detection technologies will result in accessible and affordable tests for the diagnosis of STI in resource-poor settings in the next few years. Among them are duplex antigen tests that can be used for STI syndromes and rapid lateral flow tests that can be used with oral fluid. Molecular tests providing sample-in, answer-out convenience or simple isothermal nucleic acid amplification technologies that can give an answer in 15-60 min with no special equipment will provide unprecedented test performance with ease of use. Financing mechanisms and implementation issues in resource-poor settings are important areas to address to ensure the full impact of new technologies.

Competing interests None declared.

Provenance and peer review Commissioned; externally peer reviewed.

\section{REFERENCES}

1. Aledort JE, Ronald A, Rafael ME, et al. Reducing the burden of sexually transmitted infections in resource-limited settings: the role of improved diagnostics. Nature 2006;444(Suppl 1):59-72

2. Peeling RW. Utilisation of rapid tests for sexually transmitted infections: promises and challenges. Open Infect Dis J 2009;3:156-63.

3. Castro AR, Esfandiari J, Kumar $\mathrm{S}$, et al. Novel point-of-care test for simultaneous detection of nontreponemal and treponemal antibodies in patients with syphilis. J Clin Microbiol 2010;48:4615-19.

4. Mahilum-Tapay L, Laitila V, Wawrzyniak JJ, et al. New point of care Chlamydia Rapid Test - bridging the gap between diagnosis and treatment: performance evaluation study. BMJ 2007;335:1190-4.

5. Sorger PK. Microfluidics closes in on point-of-care assays. Nat Biotechnol 2008;26:1345-6.

6. Van Rie A, Page-Shipp L, Scott L, et al. Xpert(®) MTB/RIF for point-of-care diagnosis of TB in high-HIV burden, resource-limited countries: hype or hope? Expert Rev Mol Diagn 2010;10:937-46.

7. Piepenburg $\mathbf{0}$, Williams $\mathbf{C H}$, Stemple DL, et al. DNA detection using recombination proteins. PLOS Biol 2006:4:e204.

8. Mahmoudian L, Kaji N, Tokeshi M, et al. Rolling circle amplification and circle-tocircle amplification of a specific gene integrated with electrophoretic analysis on a single chip. Anal Chem 2008;80:2483-90.

9. Pang T, Peeling RW. Diagnostic tests for infectious diseases in the developing world: two sides of the coin. Trans R Soc Trop Med Hyg 2007:101:856-7.

10. World Bank. World Development Report 2004: Making Services Work for Poor People. New York: Oxford University Press for the World Bank, 2004.

11. Peeling RW, Ronald A. Diagnostic challenges of sexually transmitted infections in resource-limited settings. Future Microbiol 2009;4:1271-82. 